\title{
Water self-diffusivity confined in graphene nanogap using molecular dynamics simulations
}

M. Moulod, and G. Hwang

Citation: Journal of Applied Physics 120, 194302 (2016); doi: 10.1063/1.4967797

View online: https://doi.org/10.1063/1.4967797

View Table of Contents: http://aip.scitation.org/toc/jap/120/19

Published by the American Institute of Physics

\section{Articles you may be interested in}

A general purpose model for the condensed phases of water: TIP4P/2005

The Journal of Chemical Physics 123, 234505 (2005); 10.1063/1.2121687

Labyrinthine water flow across multilayer graphene-based membranes: Molecular dynamics versus continuum predictions

The Journal of Chemical Physics 144, 234701 (2016); 10.1063/1.4953685

Comparison of simple potential functions for simulating liquid water

The Journal of Chemical Physics 79, 926 (1983); 10.1063/1.445869

Simulations of structural and dynamic anisotropy in nano-confined water between parallel graphite plates

The Journal of Chemical Physics 137, 184703 (2012); 10.1063/1.4763984

Carbon membranes for efficient water-ethanol separation

The Journal of Chemical Physics 145, 124708 (2016); 10.1063/1.4963098

Flow of quasi-two dimensional water in graphene channels

The Journal of Chemical Physics 148, 064702 (2018); 10.1063/1.5017491

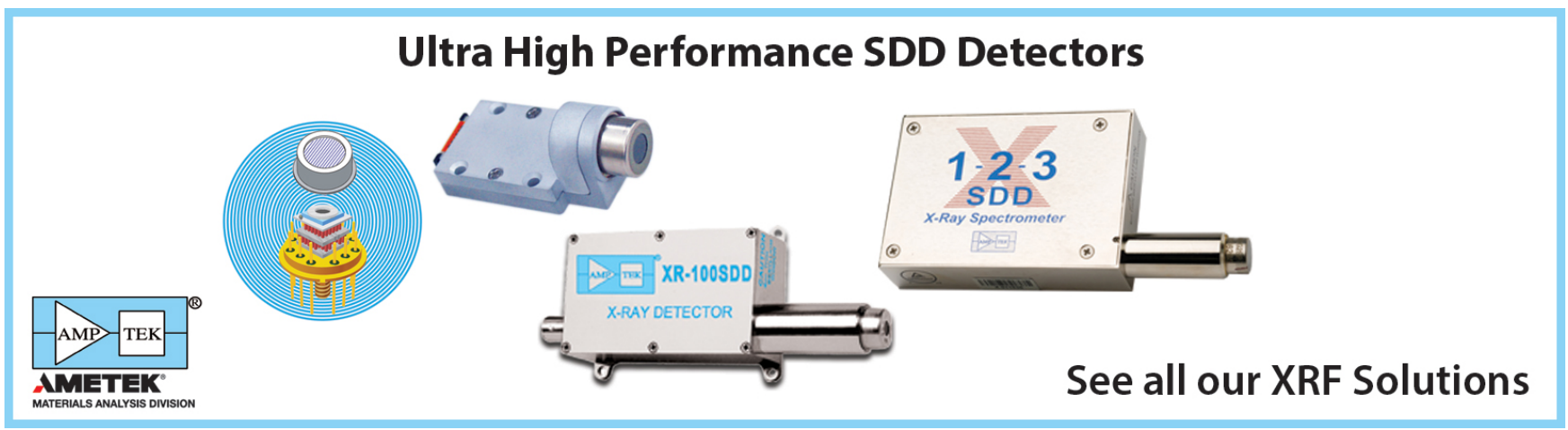




\title{
Water self-diffusivity confined in graphene nanogap using molecular dynamics simulations
}

\author{
M. Moulod and G. Hwang ${ }^{\text {a) }}$ \\ Mechanical Engineering Department, Wichita State University, Wichita, Kansas 67260, USA
}

(Received 30 September 2016; accepted 2 November 2016; published online 17 November 2016)

\begin{abstract}
Fundamental understanding of water confined in graphene is crucial to optimally design and operate sustainable energy, water desalination, and bio-medical systems. However, the current understanding predominantly remains in the static properties near the graphene surfaces. In this paper, a key water transport property, i.e., self-diffusivity, is examined under confinement by various graphene nanogap sizes $\left(L_{z}=0.7-4.17 \mathrm{~nm}\right)$, using molecular dynamics simulations with various graphene-water interatomic potentials (Simple Point Charge (SPC/E) and TIP3P water models). It is found that the water self-diffusivity nearly linearly decreases as the graphene-water interatomic potential energy increases at a given nanogap size. It also decreases as the graphene nanogap size decreases down to $L_{z}=1.34 \mathrm{~nm}$; however, it shows the peak water self-diffusivity at $L_{z}=0.8 \mathrm{~nm}$ and then continues to decrease. The peak water self-diffusivity is related to the significant change of the overlapping surface force, and associated, nonlinear local water density distribution. The in-plane water selfdiffusivity is higher up to nearly an order of magnitude than that of the out-of-plane due to the geometrical confinement effect by the graphene nanogap. The obtained results provide a roadmap to fundamentally understand the water transport properties in the graphene geometries and surface interactions. Published by AIP Publishing. [http://dx.doi.org/10.1063/1.4967797]
\end{abstract}

\section{INTRODUCTION}

Graphene is one of the promising materials which is used in various energy, environmental, and biomedical applications, including fuel cells, ${ }^{1-11}$ super capacitors, ${ }^{12-16}$ carbon capture, ${ }^{17,18}$ water desalination, ${ }^{19}$ sensors, ${ }^{20-23}$ drug delivery, $^{24-26}$ and biomedical systems. ${ }^{27-29}$ Especially, water behaviors at graphene interfaces have received great interests due to the importance of such ubiquitous working fluid in such systems. ${ }^{1-7,9-11,19,24-30}$ For desired material design and operating conditions, fundamental understanding of the water behaviors in the presence of graphene is essential. Recent studies have shown that the static/dynamic water behaviors confined in the graphene are significantly different from those of the bulk water, including water wetting, density/structure, ${ }^{31,32}$ self-diffusivity, ${ }^{33-36}$ and viscosity, ${ }^{36,37}$ due to different hydrogen bond network of water, ${ }^{38}$ formation of helical ice-like structure, ${ }^{39}$ ordered ice-like nanotube, ${ }^{40}$ and abnormally fast water transport ${ }^{41}$ near the water-carbon interface.

To fundamentally understand the nanoscale water behaviors, the molecular dynamics simulation (MDS) complements experimental studies due to flexible controls for the simulation setup and operation conditions. ${ }^{42,43}$ One of the key inputs for the MDS is interatomic interactions among the water-water ${ }^{44-47}$ and water-carbon. ${ }^{34,35,48-51}$ However, a challenge is the lack of universally accepted interatomic potential of water-carbon interactions, resulting in poor understanding of the water behavior in graphene nanogap (especially at the interfaces). A typical approach to model the water-carbon interatomic interactions (non-bonded) is to

\footnotetext{
a) Author to whom correspondence should be addressed. Electronic mail: Gisuk.Hwang@wichita.edu.
}

employ the pairwise additive Lennard Jones (LJ) potential given as

$$
\varphi_{i j}=4 \varepsilon_{i j}\left\{\left(\frac{\sigma_{i j}}{r_{i j}}\right)^{12}-\left(\frac{\sigma_{i j}}{r_{i j}}\right)^{6}\right\},
$$

where $\varepsilon_{i j}$ is the potential well depth, $2^{1 / 6} \sigma_{i j}$ is the position of the potential well, and $r_{i j}$ is the separation distance between a pair of atoms $i$ and $j$, i.e., carbon-oxygen or carbonhydrogen.

The various carbon-water potentials have been developed using the empirical approach ${ }^{41,52-54}$ and $a b$ initio calculations, ${ }^{55}$ while numerous studies have adopted the potential parameters ${ }^{31,38-40}$ based on the combination rule using the existing C-C, O-O, and $\mathrm{H}-\mathrm{H}$ interactions. ${ }^{42,49}$ For empirical approach, Markovic et al. ${ }^{52}$ have employed $\sigma_{\mathrm{C}-\mathrm{O}}=3.19 \AA$, $\varepsilon_{\mathrm{C}-\mathrm{O}}$ $=0.0747 \mathrm{kcal} / \mathrm{mol}, \quad \sigma_{\mathrm{C}-\mathrm{H}}=2.82 \AA, \quad \varepsilon_{\mathrm{C}-\mathrm{H}}=0.0483 \mathrm{kcal} / \mathrm{mol}$, showing that this model has a good agreement to the results of scattering intensities and angular average velocities. Hummer et al. ${ }^{41}$ have employed $\varepsilon_{\mathrm{C}-\mathrm{O}}=0.47847 \mathrm{~kJ} / \mathrm{mol}$ and $\sigma_{\mathrm{C}-\mathrm{O}}$ $=3.275 \AA$ to study the spontaneous water transport in carbon nanotube $(\mathrm{CNT})$. They have also considered a reduced interaction with $\varepsilon_{\mathrm{C}-\mathrm{O}}=0.2703 \mathrm{~kJ} / \mathrm{mol}$ and $\sigma_{\mathrm{C}-\mathrm{O}}=3.4138 \AA$ to explore the role of the carbon-water interaction on water transport. Werder et al $^{53}$ have used the $\varepsilon_{\mathrm{C}-\mathrm{O}}=0.3135 \mathrm{~kJ} / \mathrm{mol}$ and $\sigma_{\mathrm{C}-\mathrm{O}}$ $=3.190 \AA$ obtained from the experimental data ${ }^{56}$ to best predict the water contact angle in CNT and to best fit into the experimentally observed water contact angle of $86^{\circ}$ on graphite using $\varepsilon_{\mathrm{C}-\mathrm{O}}=0.0937 \mathrm{kcal} / \mathrm{mol}$ and $\sigma_{\mathrm{C}-\mathrm{O}}=3.190 \AA$ with the Simple Point Charge (SPC/E) water model ${ }^{49}$ and the binding energy of $-6.33 \mathrm{~kJ} / \mathrm{mol}^{57}$ For the $a b$ initio calculation, $\mathrm{Wu}$ and Aluru ${ }^{55}$ have studied the effective potential and reported $\varepsilon_{\mathrm{C}-\mathrm{O}}=0.1039 \mathrm{kcal} / \mathrm{mol}, \sigma_{\mathrm{C}-\mathrm{O}}=3.372 \AA$, and 
$\varepsilon_{\mathrm{C}-\mathrm{H}}=0.0256 \mathrm{kcal} / \mathrm{mol}, \sigma_{\mathrm{C}-\mathrm{H}}=2.640 \AA$, fitting into the previous modeling work using the density functional theorysymmetry adapted perturbation theory (DFT-SAPT), ${ }^{58}$ which have agreed to the water contact angle and radial breathing mode (RBM) frequency. Several representative carbon-water interactions are summarized in Table I, which are used in this study. Further detailed discussions about water behaviors on CNT and graphite are found in literature. ${ }^{49,59}$

These potentials have also been used for dynamic water property (water self-diffusivity) on the CNT and graphene nanogap; however, the reported water self-diffusivities under confinement of CNT and/or graphene nanogap have been contradictory. Marti et al..$^{34}$ have employed MDS with a flexible Simple Point Charge (SPC) water model and LJ potentials for water-graphene interactions of both $\mathrm{C}-\mathrm{O}$ and $\mathrm{C}-\mathrm{H}$ confined in graphene nanogap, $L_{z}=3.1 \mathrm{~nm}$, to study the water self-diffusivity at $298 \mathrm{~K}<T<673 \mathrm{~K}$. They have observed that the water confined in the graphene nanogap has higher water diffusivity than that of unconfined water, and the water self-diffusivity near the interface is lower than the central, bulk-like regions. They have concluded that the enhanced water self-diffusivity is primarily related to the fact that it has the larger in-plane ( $x$ - and $y$-directions) water self-diffusivity than out-of-plane (z-direction) one due to the geometrical confinement by the graphene nanogap. ${ }^{35}$ Similarly, Rana and Chandra have shown the moderate increase in the water self-diffusivity of the first solvation layer near the graphene sheet using SPC/E water model. ${ }^{42}$ They have concluded that this moderate increase is related to the high local water density in the first solvation layer near the graphene sheet. ${ }^{31}$ Kim et al. have examined the water diffusivity confined in the graphene nanogap, $L_{z}=1.4 \mathrm{~nm}$, showing the significant increase although the biphilic nanogap (one side is hydrophobic and the other side is hydrophilic) decreases the water self-diffusivity. ${ }^{60}$ The enhanced water self-diffusivity is also observed in the highly confined environment, i.e., one-dimensional water molecular chains by CNT, leading to the early phase transition, and this results in the fast transport properties due to limited hydrogen bond network. ${ }^{41}$ On the contrary, some of the previous studies have shown a reduced water self-diffusivity confined in both graphene nanogap and CNT. Choudhury and Pettitt have reported that both the translational and reorientational water

TABLE I. Summary of carbon-water interatomic potentials used.

\begin{tabular}{lccccc}
\hline \hline Case & $\sigma_{\mathrm{C}-\mathrm{O},} \AA$ & $\varepsilon_{\mathrm{C}-\mathrm{O}}, \mathrm{kcal} / \mathrm{mol}$ & $\sigma_{\mathrm{C}-\mathrm{H},} \AA$ & $\varepsilon_{\mathrm{C}-\mathrm{H}}, \mathrm{kcal} / \mathrm{mol}$ & Ref./symbol $^{\mathrm{a}}$ \\
\hline 1 & 3.19 & 0.0478 & $\ldots$ & $\ldots$ & $54 \boldsymbol{\square}$ \\
2 & 3.19 & 0.0937 & $\ldots$ & $\ldots$ & $49 \square$ \\
3 & 3.275 & 0.1143 & $\ldots$ & $\ldots$ & $41 \bigcirc$ \\
4 & 3.126 & 0.1646 & 2.447 & 0.0246 & $55 \Sigma$ \\
5 & 3.19 & 0.0935 & 2.82 & 0.0605 & $52 \star$ \\
6 & 3.28 & 0.0930 & 2.81 & 0.0308 & $38 \boldsymbol{\Delta}$ \\
7 & 3.372 & 0.1039 & 2.640 & 0.0256 & $55 \triangle$ \\
8 & 3.43 & 0.1164 & 2.685 & 0.0263 & $55 \square$ \\
9 & 3.436 & 0.0850 & 2.69 & 0.0383 & $55 \bigcirc$ \\
10 & 3.19 & 0.1349 & $\ldots$ & $\ldots$ & $49 \bullet$ \\
\hline \hline
\end{tabular}

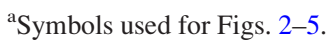

mobilities confined in the graphene nanogap significantly reduced at $L_{z}=1.3 \mathrm{~nm} .{ }^{61}$ Mashl et al. ${ }^{62}$ have examined the water confined in CNT using a rigid SPC model at ambient temperature and pressure, showing that the mobility of water (ice-like) significantly reduces due to the phase transition. Liu et $a l^{63}$ have also showed that the water self-diffusivity in CNT is much lower than those of the bulk, and the diffusivity also decreases with decreasing CNT diameter. Hanasaki and Nakatani ${ }^{64}$ have shown the lower water self-diffusivity, which is related to the nonlinear hydrogen bond lifetime with respect to the CNT diameter. In fact, Cicero et al. ${ }^{36}$ have examined that the water-model-dependent self-diffusivity (flexible and rigid SPC/E), showing the flexible SPC/E for a higher diffusivity than that of bulk and the rigid water model for a lower diffusivity.

The aforementioned contentious results on the water self-diffusivities are primarily related to the fact that the previous studies have used the different carbon-water interaction parameters. The different interaction parameters change the water local density profiles near the CNT and graphene nanogap surfaces, which have in turn resulted in the different water self-diffusivities. In this paper, we extensively use the available water-graphene models in literature ${ }^{49,55}$ aiming at articulating dynamic water behaviors confined in graphene, i.e., water self-diffusivity, local density distributions, under the various graphene geometries (nanogap sizes) and watercarbon surface energies, employing classical MD simulation.

\section{METHODOLOGY}

The water was simulated in the graphene nanogap with the various nanogap sizes, $L_{z}=0.7-4.17 \mathrm{~nm}$, while the periodic boundary conditions were used for $x$ - and $y$-axes using $L_{x}=29.47 \mathrm{~nm}$ and $L_{y}=29.77 \mathrm{~nm}$ as illustrated in Fig. 1, and SPC/E and TIP3P water models with a rigid graphene were used. ${ }^{36,44,49,65}$ Although the accuracy of the predicted water self-diffusivity within the graphene nanogap may not be ideal, especially for TIP3P model, we used SPC/E and TIP3P water models because they are extensively studied in literature. $^{36,39,43,44,47,49,55,59,60,66,67}$ Also, the focus of this study is to elucidate the role of the interaction parameters on the water self-diffusivity using MDS. Thus, the predicted results using TIP3P model provide a reasonable insight into the atomic scale water behaviors within the graphene nanogap without losing a generality.

The water molecules were treated as a rigid structure using SHAKE algorithm ${ }^{68}$ for both water models. Initially, the water molecules were filled within nanogap, and the number of water molecules were adjusted for a constant density, $N=36-1,020$ for different nanogap sizes, $L_{z}=0.7-4.17 \mathrm{~nm}$. For the graphene nanogap, the water-graphene interfacial region was not occupied with water molecules which make it challenging to calculate the water density in the graphene nanogap. In previous studies, they excluded water unoccupied volume from the nanogap, i.e., subtracting $2.5 \AA$ (for each surface) from the carbon nanogap. ${ }^{34,36,42}$

In this study, we used $2.7 \AA$ for the water density, since the "unoccupied region" varied between 2.6 and $2.8 \AA$ depending on the carbon-water interaction parameters. For 


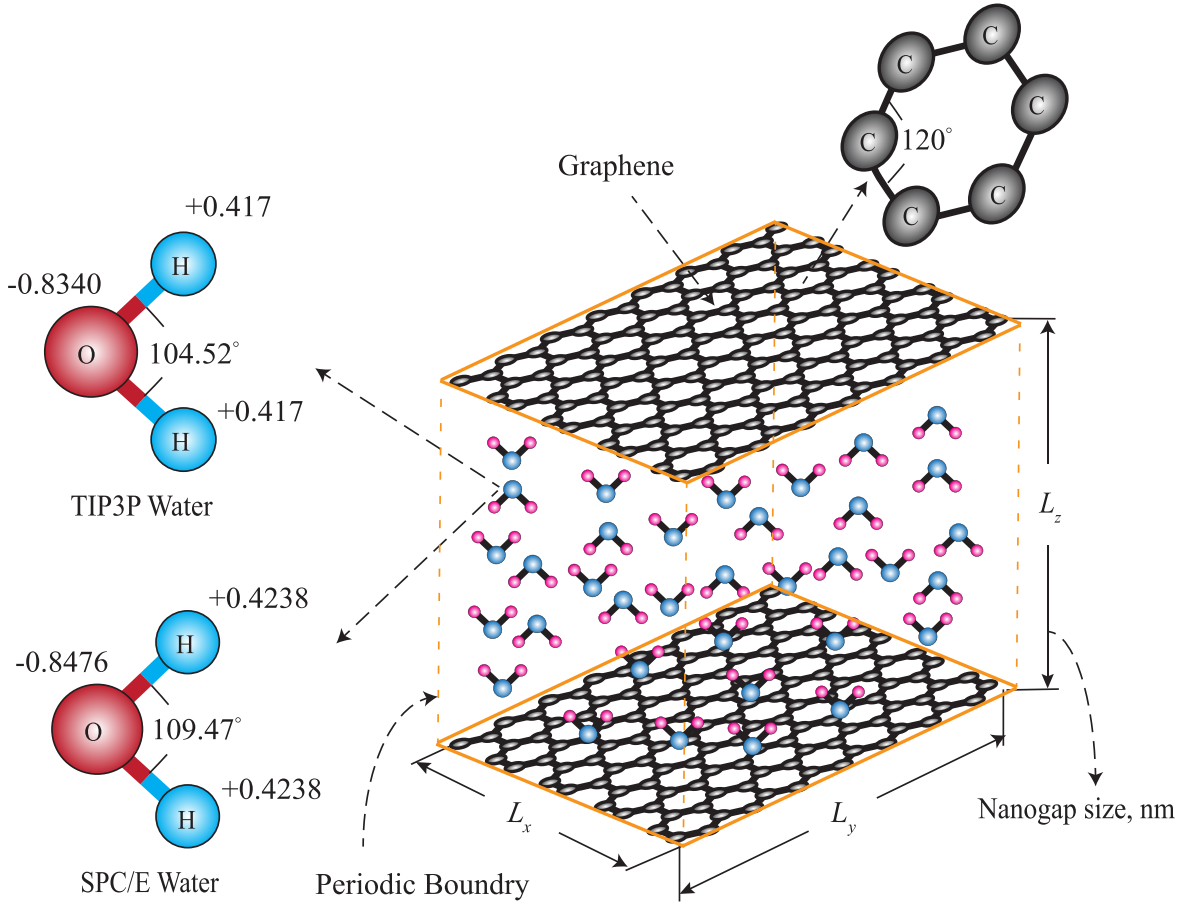

FIG. 1. A schematic of water confined in graphene nanogap, including the details of the water and graphene models. Periodic boundary for $x$ and $y$-coordinates, charges, and angles for the water molecules are also shown.

all the MDS, a Large-scale Atomic/Molecular Massively Parallel Simulator (LAMMPS) was used. ${ }^{69}$ In order to simulate water in graphene nanogap, different interactions were used for carbon-oxygen and carbon-hydrogen of water molecules as summarized in Table I. For water-water and water-graphene intra- and interatomic potentials, the harmonic bond and angle model were used. The long-range electrostatic force was calculated using the pairwise additive method, where the damping coefficient, $\alpha=0.2 \AA^{-1}$, and cutoff $r_{c}=1.2 \mathrm{~nm}$ were used. ${ }^{70}$ The pair-wise long range interaction has been successfully used for water similarly confined in the nanogap structure, where the predicted water self-diffusivities show a reasonable agreement with the experimental results. ${ }^{71}$ Thus, we adopted this as a valid approach in this study.

Mean squared displacement (MSD) was used to calculate diffusivity of water confined in different nanogaps at $T=298.15 \mathrm{~K}$ given as ${ }^{72}$

for 3D (all $x$-, $y$-, and $z$-directions)

$$
\langle D\rangle=\lim _{n \rightarrow \infty} \frac{1}{6 N t}\left\langle\sum_{j=1}^{N}\left[r_{j}(t)-r_{j}(0)\right]^{2}\right\rangle,
$$

for 2D (in plane, $x$-, $y$-directions only)

$$
\left\langle D_{x y}\right\rangle=\lim _{n \rightarrow \infty} \frac{1}{4 N t}\left\langle\sum_{j=1}^{N}\left[r_{j}(t)-r_{j}(0)\right]^{2}\right\rangle,
$$

for 1D (out-of-plane, $z$-direction only)

$$
\left\langle D_{z}\right\rangle=\lim _{n \rightarrow \infty} \frac{1}{2 N t}\left\langle\sum_{j=1}^{N}\left[r_{j}(t)-r_{j}(0)\right]^{2}\right\rangle,
$$

where $\langle D\rangle$ is the water self-diffusivity, $r_{j}(t)$ is the position of the center of mass of the molecule $j$ at a time $t, N$ is the total number of molecules, and \langle\rangle shows an ensemble average, and the center of water mass was used to calculate the water self-diffusivity. For Eq. (3), only $x$ and $y$ coordinates are used for the in-plane water self-diffusivity, i.e., $\left\langle D_{x y}\right\rangle$, while for Eq. (4), only $z$ coordinate is used for the out-of-plane water self-diffusivity, i.e., $\left\langle D_{z}\right\rangle$.

The Number, Volume, and Temperature (NVT) ensemble is used to reach equilibrium for $2 \mathrm{~ns}$ at $T=298.15 \mathrm{~K}$, followed by the Number, Volume, and Energy (NVE) ensemble for 4.1 and $2 \mathrm{~ns}$ to calculate the water diffusivity in bulk water and graphene nanogap, respectively. In bulk water, the $N=906$ is used for the density of $\rho=998 \mathrm{~kg} / \mathrm{m}^{3}$, and the simulation results are compared with the previous study within a few percent error. ${ }^{47}$ The simulations at given temperature and nanogap sizes are repeated 5 times using different initial velocities for the reproducibility check. The above-mentioned parameters are summarized in Table II.

\section{RESULTS AND DISCUSSION}

\section{A. Water self-diffusivity vs water-graphene interatomic potentials}

Fig. 2 shows the water self-diffusivity of TIP3P and $\mathrm{SPC} / \mathrm{E}$ water models as a function of total interatomic

TABLE II. Ensembles and parameters used in simulations.

\begin{tabular}{lc}
\hline \hline Parameters & Types/magnitude \\
\hline Water models & SPC/E and TIP3P original ${ }^{44}$ \\
Temperature & $298.15 \mathrm{~K}$, Nose-Hoover Thermostat \\
Water density & $998 \mathrm{~kg} / \mathrm{m}^{3}$ for both water models \\
Structure control algorithms & Harmonic and SHAKE algorithm \\
Cut off length, $r_{c}$ & $1.2 \mathrm{~nm}$ \\
Nanogap sizes $\left(L_{x}, L_{y}\right.$, and $\left.L_{z}\right)$ & $2.95 \mathrm{~nm}, 2.98$, and $0.7-4.17 \mathrm{~nm}$ \\
Number of water molecules & $36-1020$ \\
Ensembles & NVT (equilibrium) $2 \mathrm{~ns}$ \\
& and NVE (properties) $2.0-4.1 \mathrm{~ns}$ \\
\hline \hline
\end{tabular}


potential energies between $\mathrm{C}-\mathrm{O}$ and $\mathrm{C}-\mathrm{H}$, i.e., $\varepsilon_{\mathrm{C}-\mathrm{O}}+\varepsilon_{\mathrm{C}-\mathrm{H}}$, for the nanogap size of $L_{z}=2.63 \mathrm{~nm}$. Note that in some of the interaction parameters between the water and graphene, $\mathrm{C}$-H is zero, i.e., $\varepsilon_{\mathrm{C}-\mathrm{H}}=0 .{ }^{49,55}$ The water self-diffusivity nearly-linearly decreases with the increasing surface energy, while all the diffusivities remain lower than that of the bulk water (only $\sim 70 \%$ of the bulk water). The increasing surface energy leads to a strong water-graphene interaction (water molecules are attractive to the graphene surface), resulting in the poor mobility of the water near the graphene, especially in the $z$ direction. The calculated linear curve fit based on the obtained data is given as

$$
\left\langle D_{\mathrm{H}_{2} \mathrm{O}}\right\rangle=a\left(\varepsilon_{\mathrm{C}-\mathrm{O}}+\varepsilon_{\mathrm{C}-\mathrm{H}}\right)+b
$$

where $\left\langle D_{\mathrm{H}_{2} \mathrm{O}}\right\rangle$ is the water self-diffusivity in $\mathrm{m}^{2} / \mathrm{s}$, and $\varepsilon_{\mathrm{C}-\mathrm{O}}$ is the carbon-oxygen $\mathrm{LJ}$ potential energy in $\mathrm{kcal} / \mathrm{mol}, a$ and $b$ are the fitting parameters in $\left[\mathrm{m}^{2} / \mathrm{s}\right] /[\mathrm{kcal} / \mathrm{mol}]$ and $\mathrm{m}^{2} / \mathrm{s}$, respectively, and $a=-0.867 \times 10^{-9}$ and $b=4.258 \times 10^{-9}$ are found for the TIP3P in Fig. 2(a). Note that there are a few data with a discrepancy from the curve fit, and this is mainly caused by the fact that the results are based on the surface interactions both between $\mathrm{C}-\mathrm{O}$ and $\mathrm{C}-\mathrm{H}$. Note that no strong correlation between the water self-diffusivity and $\sigma_{\mathrm{C}-\mathrm{O}}$ or $\sigma_{\mathrm{C}-\mathrm{H}}$ is found. Furthermore, the similar results are found for the different nanogap sizes, and we report the results only for $L_{z}=2.63 \mathrm{~nm}$ here, for a representative case. The similar water diffusivity trends are also found as shown in Fig. 2(b) for SPC/E water model at the same nanogap size, and the fitting parameters are $a=-1.366 \times 10^{-9}$ and $b=2.209 \times 10^{-9}$, in Eq. (5). Note that the water self-diffusivity is still expected to be lower than that of the bulk water, even with the virtually no water-graphene surface interaction energy, $\varepsilon_{\mathrm{C}-\mathrm{O}}+\varepsilon_{\mathrm{C}-\mathrm{H}}=0$ (when it is extrapolated to the zero surface interaction energy), and this translates into the fact that the "structural" confinement by the nanogap reduces the water transport $\sim 20 \%$. This has been similarly observed in the water contact angle, i.e., the water contact angle linearly decreases with the increase in the surface energy. ${ }^{49}$

(a)

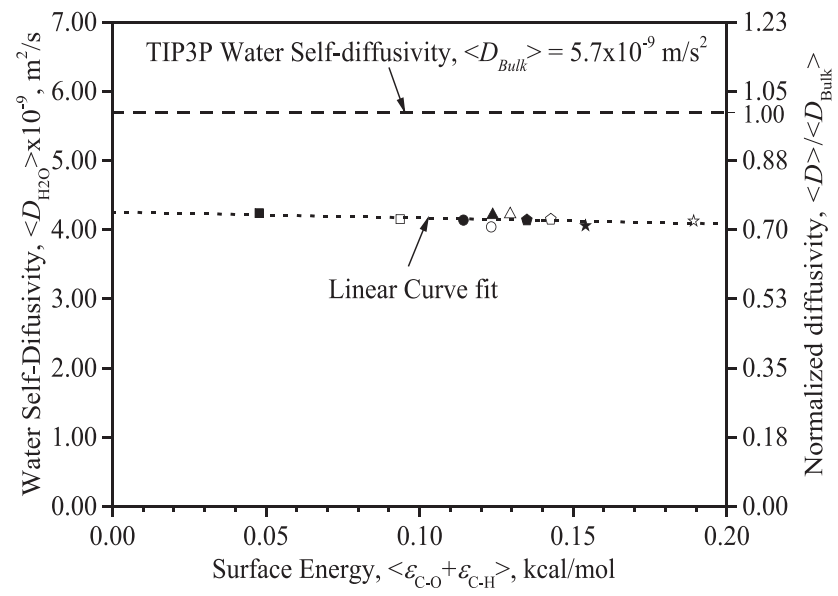

\section{B. Water self-diffusivity vs. nanogap sizes}

Fig. 3 shows the predicted water self-diffusivities (total, in-plane, and out-of-plane diffusivities) as a function of nanogap size, $L_{z}=0.7-4.17 \mathrm{~nm}$, using $\sigma_{\mathrm{C}-\mathrm{O}}=3.19 \AA$ and $\varepsilon_{\mathrm{C}-\mathrm{O}}$ $=0.0937 \mathrm{kcal} / \mathrm{mol}\left(\varepsilon_{\mathrm{C}-\mathrm{H}}=0\right)$ for both the TIP3P and SPC $/ \mathrm{E}$ water models, considering one of the representative $\mathrm{C}-\mathrm{O}$ potentials based on the reasonable water contact angle on graphite. ${ }^{49}$ For both water models, the water self-diffusivity for all the nanogap sizes is smaller than that of the bulk water due to the geometrical confinement effect. The water selfdiffusivity generally decreases with the decrease in the nanogap size, and this indicates that the strong water-graphene surface interactions significantly reduce the water transport. As the nanogap size increases, the water self-diffusivity gradually increases toward the bulk water self-diffusivity. Note that when the nanogap size is smaller than $2 \mathrm{~nm}$, i.e., $L_{z}<2 \mathrm{~nm}$, it has a significant surface force overlap across the nanogap (the cut off radius $r_{c}$ is $1.2 \mathrm{~nm}$ ), which results in the further water transport reduction, i.e., a significant water diffusivity reduction at $L_{z}<0.8 \mathrm{~nm}$. Interestingly, it shows a peak at $L_{z}=0.8 \mathrm{~nm}$, and this is related to the noticeable changes in local water density distribution across the nanogap at $L_{z}=0.7-1.34 \mathrm{~nm}$, and further discussions will be given below. For all the nanogap size, the in-plane water diffusivity, $\left\langle D_{x y}\right\rangle$, is much larger (up to the order of magnitude) than that of the out-plane water diffusivity, $\left\langle D_{z}\right\rangle$, due to the geometrical confinement given in only out-of-plane direction (Fig. 3). For the small nanogap, $L_{z}<1.3 \mathrm{~nm}$, the total water self-diffusivity, $\langle D\rangle$, is dominated by the in-plane water diffusivity, $\left\langle D_{x y}\right\rangle$, while it shows minimal contributions from the negligibly small out-of-plane diffusivity, $\left\langle D_{z}\right\rangle$ due to the significant geometrical confinement. The $\left\langle D_{x y}\right\rangle$ is also larger than the bulk water self-diffusivity $\left\langle D_{b u l k}\right\rangle$, especially at smaller nanogap, and it becomes similar to $\left\langle D_{\text {bulk }}\right\rangle$ as the nanogap size increases (it becomes bulk-like behaviors as the nanogap size increases). Note that the peak of $\langle D\rangle$ at $L_{z}$ $=0.8 \mathrm{~nm}$ is related to the peak of $\left\langle D_{x y}\right\rangle$. As the nanogap size increases, the $\langle D\rangle$ increases, and this is related to the significant diffusivity enhancement of $\left\langle D_{z}\right\rangle$ due to the increased

(b)

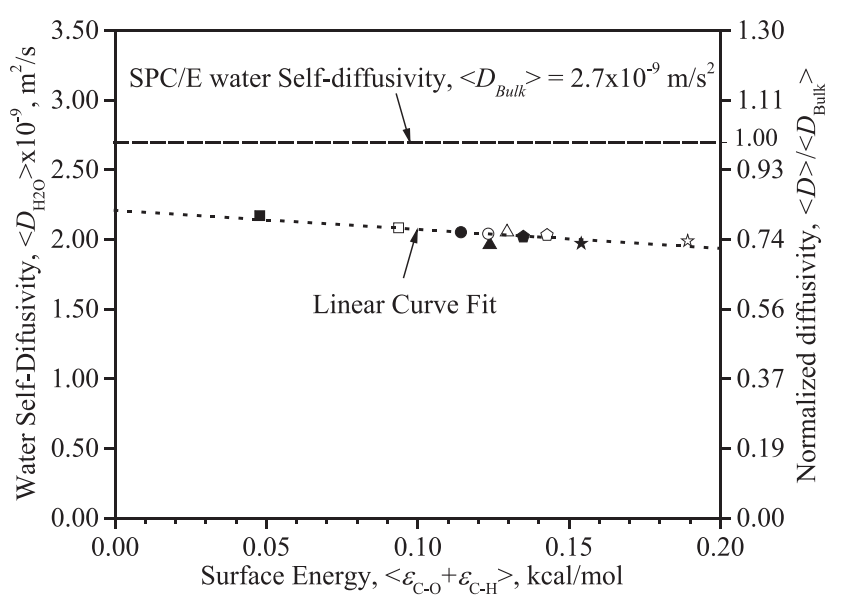

FIG. 2. Predicted water self-diffusivity as a function of the total potential interatomic energy between C-O and C-H at the nanogap size of $L_{z}=2.63$ nm and $N=614$ water molecules, for (a) TIP3P and (b) SPC/E water. A linear curve fit for the surface energy and bulk water self-diffusivity is also shown. 
(a)

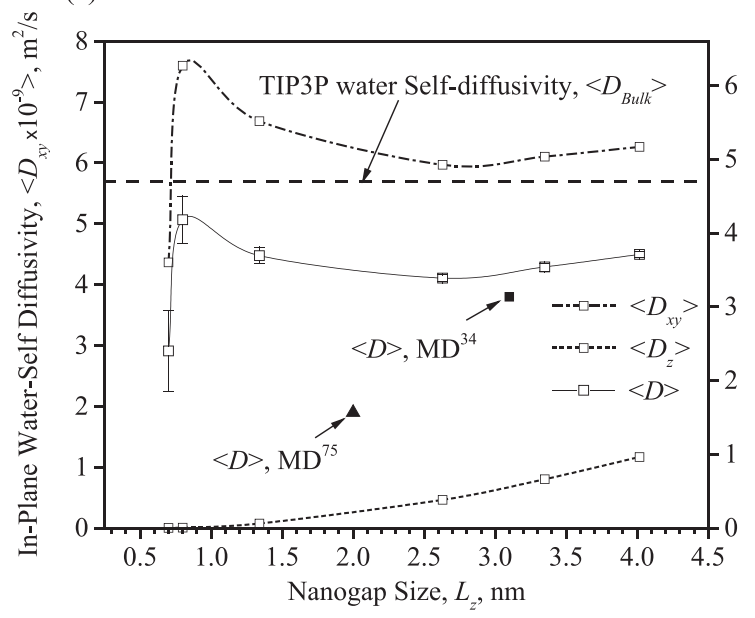

(b)

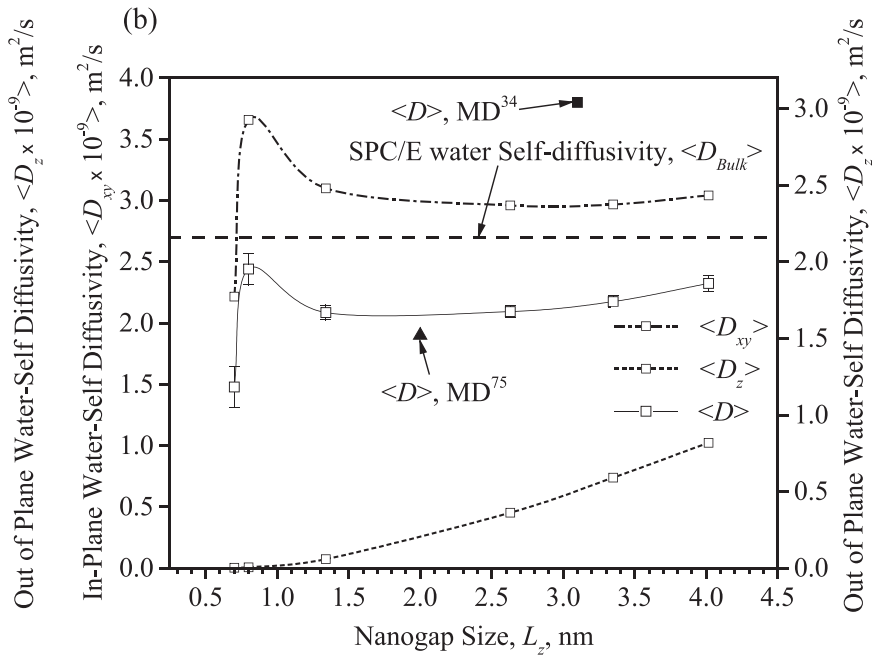

FIG. 3. Variations of average, in-plane (left $y$-axis), $\left\langle D_{x y}\right\rangle$, and out-of-plane (right $y$-axis) water self-diffusivity, $\left\langle D_{z}\right\rangle$, as a function of the nanogap size, $L_{z}=0.7$ to $4.17 \mathrm{~nm}$, using $\sigma_{\mathrm{C}-\mathrm{O}}=3.19 \AA$ and $\varepsilon_{\mathrm{C}-\mathrm{O}}=0.0937 \mathrm{kcal} / \mathrm{mol}$, for (a) TIP3P model, and (b) for SPC/E. The bulk water self-diffusivities and error bars are also shown. Other MDS results are also shown. ${ }^{34,74}$

bulk regions (central regions of nanogap, where the water transport is unhindered by the surface interactions), while the $\left\langle D_{x y}\right\rangle$ does not change at $L_{z}>1.3 \mathrm{~nm}$. This reveals that the origin of the $\langle D\rangle$ is caused by the different contributions of $\left\langle D_{x y}\right\rangle$ and $\left\langle D_{z}\right\rangle$ associated with the different level of geometrical confinement, i.e., nanogap size. Note that the smallest gap size, i.e., $L_{z}=0.7 \mathrm{~nm}$, has only 37 water molecules, resulting in the large fluctuations because of low statistics data, and 5 simulations with the different initial velocities are performed to report the average value with error bars.

Our results are compared to other MDS results in the literature for validation as shown in Fig. 3. Marti et al. have shown that the water self-diffusivity in confined nanogap size of $L_{z}=3.1 \mathrm{~nm}$ at $T=300 \mathrm{~K}$ is $\langle D\rangle=3.8 \times 10^{-9} \mathrm{~m}^{2} / \mathrm{s}$ using flexible SPC model, which agree TIP3P results of this study, ${ }^{34}$ and Zokaie and Foroutan have shown $\langle D\rangle=1.9 \times 10^{-9} \mathrm{~m}^{2} / \mathrm{s}$ for $L_{z}=2.0 \mathrm{~nm}$ at $T=300 \mathrm{~K}$ using the TIP4P/Ice water model, ${ }^{74}$ which agrees SPC/E results of this study.

In fact, the discrepancy is related to the different water models and water-carbon models used by Marti et al. (flexible SPC with $\sigma_{\mathrm{C}-\mathrm{O}}=3.28 \AA, \sigma_{\mathrm{C}-\mathrm{H}}=2.81 \AA$, $\varepsilon_{\mathrm{C}-\mathrm{O}}=0.0929 \mathrm{kcal} /$ $\mathrm{mol}$, and $\varepsilon_{\mathrm{C}-\mathrm{H}}=0.0308 \mathrm{kcal} / \mathrm{mol}$ ) ${ }^{34}$ and Zokaie et al. (TIP4P/ Ice with $\sigma_{\mathrm{C}-\mathrm{O}}=3.2571 \AA$ and $\left.\varepsilon_{\mathrm{C}-\mathrm{O}}=0.1143 \mathrm{kcal} / \mathrm{mol}\right)^{74}$ than that of this study $\left(\sigma_{\mathrm{C}-\mathrm{O}}=3.19 \AA\right.$ and $\left.\varepsilon_{\mathrm{C}-\mathrm{O}}=0.0937 \mathrm{kcal} / \mathrm{mol}\right)$. Also, note that there is a lack of available experimental data in literature for direct comparison with our simulation results.

\section{Local water density vs. water-graphene interatomic potentials}

Fig. 4 shows the local water density as a function of $z$-coordinate along nanogap for the different water-graphene surface interaction energies, $\varepsilon_{\mathrm{C}-\mathrm{O}}=0.04778,0.0937$, and $0.1646 \mathrm{kcal} / \mathrm{mol}$, at $L_{z}=2.63 \mathrm{~nm}$. The confined region is defined as the distance within the cut-off, $1.2 \mathrm{~nm}$, from the graphene surface, while the bulk region is beyond the cutoff, which are shown in Fig. 4. It shows that the high surface energy attracts water molecules to the graphene surface, which in turn causes the high density, i.e., symmetric two peaks near the surfaces. Note that the local water density near the surfaces is greater than the bulk water density, and the similar behavior has been reported for the water confined in platinum nanogap. ${ }^{75}$ The first peak shows the higher density than bulk which increases with the increasing surface energy, and the second peak occurs after the first peak, where its density is closer to the bulk water density. The water local density in the different nanogap size shows similar density distributions. Note that the water local density distribution does not show a strong correlation with the $\sigma_{\mathrm{C}-\mathrm{O}}$ or $\sigma_{\mathrm{C}-\mathrm{H}}$. The peak local water density for all the interaction energies is larger than the bulk water density, and it is more pronounced for the larger interaction energy. For the larger surface energy, i.e., $\varepsilon_{\mathrm{C}-\mathrm{O}}=0.1646 \mathrm{kcal} / \mathrm{mol}$, the significant local water density near the surface leads to the second, moderate local water peak at $0.6 \mathrm{~nm}$ from the surfaces.

\section{Local water density vs nanogap sizes}

Fig. 5 shows the local water density distribution along the $z$-direction for nanogap sizes of $L_{z}=0.7,0.8$, and $1.34 \mathrm{~nm}$ using $\sigma_{\mathrm{C}-\mathrm{O}}=3.19 \AA$ and $\varepsilon_{\mathrm{C}-\mathrm{O}}=0.0937 \mathrm{kcal} / \mathrm{mol}$ for the water SPC/E model, which is considered as one of the representative water-carbon interactions in literature (the water contact angle of $86^{\circ}$ on graphite surfaces). ${ }^{49}$ Note that the local density distribution is presented as a function of normalized $z$ by the graphene nanogap size, $L_{z}$. For $L_{z}=0.7 \mathrm{~nm}$, there is only one layer of water in the center of the nanogap, and this results in significant interactions with both surfaces (large confinement effect), which in turn causes the low water self-diffusivity (Fig. 3). For $L_{z}=0.8 \mathrm{~nm}$, it begins to have two distinct layers of water; however, the distance between the two density peak points is very small, while the water molecules still significantly interact with both surfaces. These simultaneous interactions with both surfaces moderately balance out because force directions are opposite, which results in the weaker net surface force than that of $L_{z}=0.7 \mathrm{~nm}$. This weaker surface force in turn allows for the increased water mobility and self- 

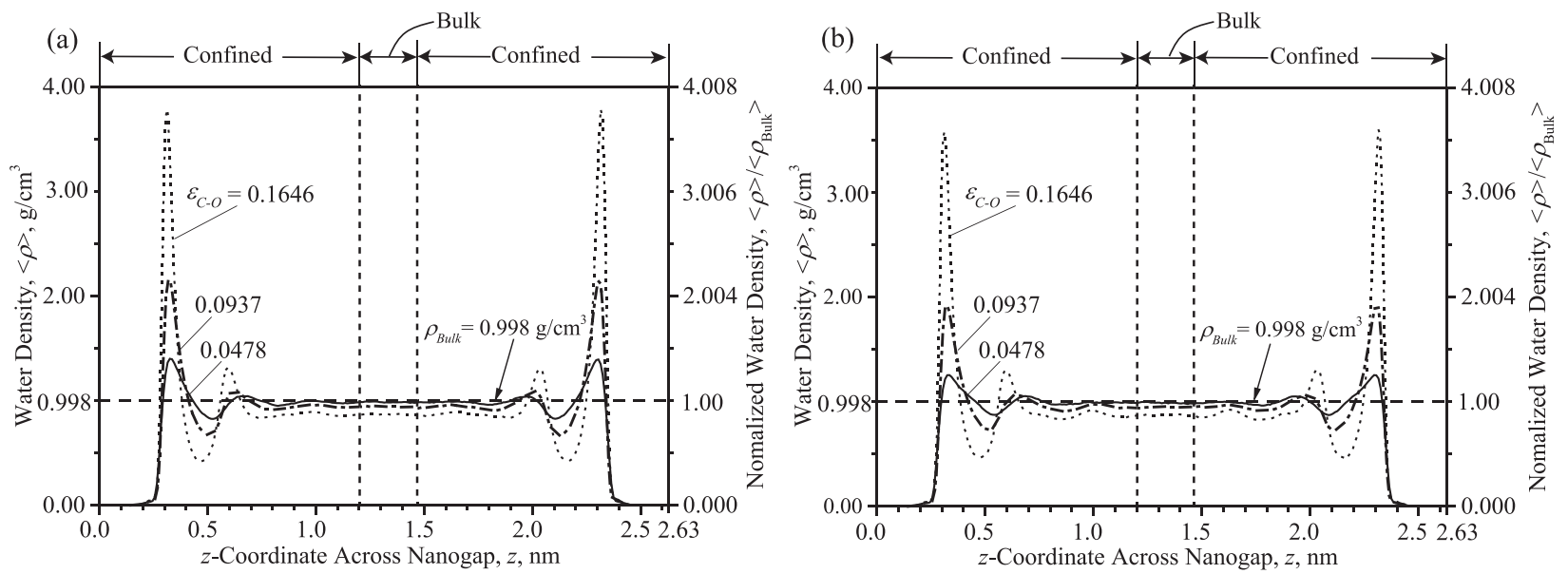

FIG. 4. Predicted local water density across the $z$-direction as a function of the C-O interatomic potential energy at $\sigma_{\mathrm{C}-\mathrm{O}}=3.19 \AA$, for the nanogap size, $L_{z}=2.63 \mathrm{~nm}$, both for (a) TIP3P, and (b) SPC/E water models. The self-diffusivity of the bulk water and relative density (normalized by the bulk water density) on right $y$-axis are also shown. Confined ( $1.2 \mathrm{~nm}$ from the graphene nanogap) and bulk regions are also shown in the $x$-axis (top).

diffusivity, showing the peak at $L_{z}=0.8 \mathrm{~nm}$ (Fig. 3). For the large nanogap, $L_{z}=1.34 \mathrm{~nm}$, the effect of surface energy on water molecules becomes stronger near the surface as the one surface dominantly attracts the water molecules, resulting in the higher local water density (and lower, local water self-diffusivity), while the water-graphene surface interaction becomes weaker in the middle of the nanogap, which in turn results in lower local density (and higher local water self-diffusivity). The similar results for the water local density in graphene nanogap have been reported in other studies. ${ }^{34,61,74,76}$ The combinational water behaviors lead to the lower self-diffusivity than that of $L_{z}=0.8 \mathrm{~nm}$, but it results in the higher than that of $L_{z}=0.7 \mathrm{~nm}$. For $L_{z}>1.34 \mathrm{~nm}$, the similar behaviors are found (not reported here), and it gradually increases the self-diffusivity as it increases the nanogap size (Fig. 3). In fact, Choudhury and Pettitt ${ }^{76}$ have studied water local density in graphene nanogap (similar size of this study, $L_{z}=0.68,0.98$, and $1.3 \mathrm{~nm}$ ), and their results are compared to our simulation results as shown in Fig. S2 in supplementary material. The local density distributions given in the

(a)

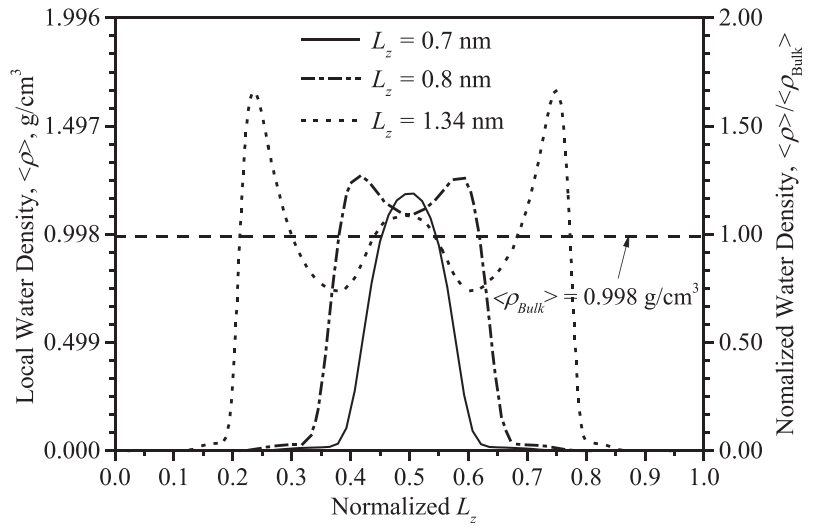

FIG. 5. Predicted local water density for different nanogap sizes, $L_{z}=0.7$, 0.8 , and $1.34 \mathrm{~nm}$, using the $\mathrm{SPC} / \mathrm{E}$ water model and $\sigma_{\mathrm{C}-\mathrm{O}}=3.19 \AA$ and $\varepsilon_{\mathrm{C}-\mathrm{O}}$ $=0.0937 \mathrm{kcal} / \mathrm{mol}$ for the water-graphene interactions. Right axis shows the normalized local and bulk water density. previous study ${ }^{76}$ are higher than those of the present study because they have used higher water-surface interaction parameters than that of the present study. Also, it has used an open nanogap system (water is freely accessible to the graphene nanogap), which in turn results in the higher local density, especially near the nanogap surfaces. Similarly, such a local water density distribution is obtained using the TIP3P water model (not shown here).

\section{CONCLUSION}

The comparative study for the water self-diffusivity confined in the various graphene nanogap sizes, $L_{z}=0.7-4.17 \mathrm{~nm}$, is performed using 10 representative water-carbon interactions with two water models utilizing the classical MDS. It is found that the water self-diffusivity nearly linearly decreases with increasing water-graphene interatomic potentials, $\varepsilon_{\mathrm{C}-\mathrm{O}}$ and $\varepsilon_{\mathrm{C}-\mathrm{H}}$, and the linear curve fits for the two water models are reported. For all nanogap sizes, the water self-diffusivity is smaller than that of the bulk water due to the nanoscale confinement effect. It is also found that the $\sigma_{\mathrm{C}-\mathrm{O}}$ (the water-graphene distance at which the intermolecular potential between the two particles is zero) does not significantly affect the water self-diffusivity. The water self-diffusivity decreases with decreasing nanogap size, with an apparent peak at $L_{z}=0.8 \mathrm{~nm}$, which is related to the local, nonlinear water distribution due to the overlapping surface forces under the confinement by the nanogap. The in-plane water self-diffusivity is significantly higher up to nearly an order of magnitude than that of the out-of-plane due to the geometrical confinement effect by the graphene nanogap, and the difference becomes smaller as the nanogap size increases (less geometrical confinement effect). The obtained results provide a roadmap to fundamentally understand the water transport properties in the graphene geometries and surface interactions.

\section{SUPPLEMENTARY MATERIAL}

See supplementary material for detailed information on graphene and water models used, and peak water density for different nanogap sizes. 


\section{ACKNOWLEDGMENTS}

This material is based upon work supported by the National Science Foundation under Award No. EPS-0903806 and matching support from the State of Kansas through the Kansas Board of Regents. This work was also partially supported by the start-up fund from the College of Engineering, Wichita State University. This work also used the Extreme Science and Engineering Discovery Environment (XSEDE), which was supported by National Science Foundation Grant No. ACI-1053575.

${ }^{1}$ L. Wang, J. Kang, J. D. Nam, J. Suhr, A. K. Prasad, and S. G. Advani, ECS Electrochem. Lett. 4, F1-F4 (2015).

${ }^{2}$ B. G. Choi, Y. S. Huh, Y. C. Park, D. H. Jung, W. H. Hong, and H. Park, Carbon 50, 5395-5402 (2012).

${ }^{3}$ Y. H. Liu, J. T. Wang, H. Q. Zhang, C. M. Ma, J. D. Liu, S. K. Cao, and X. Zhang, J. Power Sources 269, 898-911 (2014).

${ }^{4}$ T. K. Das and S. Prusty, Polym.-Plast. Technol. 52, 319-331 (2013).

${ }^{5}$ B. A. Aragaw, W. N. Su, J. Rick, and B. J. Hwang, RSC Adv. 3, 23212-23221 (2013).

${ }^{6}$ D. C. Lee, H. N. Yang, S. H. Park, and W. J. Kim, J. Membr. Sci. 452, 20-28 (2014).

${ }^{7}$ A. Iwan, M. Malinowski, and G. Pasciak, Renewable Sustainable Energy Rev. 49, 954-967 (2015)

${ }^{8}$ A. Khan, R. Asmatulu, and G. Hwang, ECS Trans. 69, 569-577 (2015).

${ }^{9}$ Z. X. Xia, S. L. Wang, L. H. Jiang, H. Sun, F. L. Qi, J. T. Jin, and G. Q. Sun, J. Mater. Chem. A 3, 1641-1648 (2015).

${ }^{10}$ B. G. Choi and H. S. Park, J. Phys. Chem. C 116, 3207-3211 (2012).

${ }^{11}$ X. Y. Zhang and Y. H. Ding, RSC Adv. 4, 44214-44222 (2014).

${ }^{12}$ K. Zhang, L. L. Zhang, X. Zhao, and J. Wu, Chem. Mater. 22, 1392-1401 (2010).

${ }^{13}$ M. F. El-Kady, V. Strong, S. Dubin, and R. B. Kaner, Science 335 , 1326-1330 (2012).

${ }^{14}$ Y. Wang, Z. Shi, Y. Huang, Y. Ma, C. Wang, M. Chen, and Y. Chen, J. Phys. Chem. C 113, 13103-13107 (2009).

${ }^{15}$ J. J. Yoo, K. Balakrishnan, J. Huang, V. Meunier, B. G. Sumpter, A. Srivastava, M. Conway, A. L. Mohana Reddy, J. Yu, and R. Vajtai, Nano Lett. 11, 1423-1427 (2011).

${ }^{16}$ A. Yu, I. Roes, A. Davies, and Z. Chen, Appl. Phys. Lett. 96, 253105 (2010).

${ }^{17}$ D. Dutta, B. C. Wood, S. Y. Bhide, K. G. Ayappa, and S. Narasimhan, J. Phys. Chem. C 118, 7741-7750 (2014).

${ }^{18}$ L. Huang, L. Zhang, Q. Shao, L. Lu, X. Lu, S. Jiang, and W. Shen, J. Phys. Chem. C 111, 11912-11920 (2007)

${ }^{19}$ D. Cohen-Tanugi and J. C. Grossman, Nano Lett. 12, 3602-3608 (2012).

${ }^{20}$ T. S. Sreeprasad, A. A. Rodriguez, J. Colston, A. Graham, E. Shishkin, V. Pallem, and V. Berry, Nano Lett. 13, 1757-1763 (2013).

${ }^{21}$ Z. Wu, X. Chen, S. Zhu, Z. Zhou, Y. Yao, W. Quan, and B. Liu, Sens. Actuators B Chem. 178, 485-493 (2013).

${ }^{22}$ F. Yavari and N. Koratkar, J. Phys. Chem. Lett. 3, 1746-1753 (2012)

${ }^{23}$ S. Basu and P. Bhattacharyya, Sens. Actuators B Chem. 173, 1-21 (2012).

${ }^{24}$ J. Liu, L. Cui, and D. Losic, Acta Biomater. 9, 9243-9257 (2013).

${ }^{25}$ S. Goenka, V. Sant, and S. Sant, J. Controlled Release 173, 75-88 (2014).

${ }^{26}$ X. Sun, Z. Liu, K. Welsher, J. T. Robinson, A. Goodwin, S. Zaric, and H. Dai, Nano Res. 1, 203-212 (2008).

${ }^{27}$ X. Zhang, J. Yin, C. Peng, W. Hu, Z. Zhu, W. Li, C. Fan, and Q. Huang, Carbon 49, 986-995 (2011).

${ }^{28}$ R. R. Nair et al., Appl. Phys. Lett. 97, 153102 (2010).

${ }^{29}$ N. Mohanty and V. Berry, Nano Lett. 8, 4469-4476 (2008).

${ }^{30}$ Y. Devrim and A. Albostan, Int. J. Hydrogen Energy 40, 15328-15335 (2015).

${ }^{31}$ H. Zhou et al., Phys. Rev. B 85, 035406 (2012).

${ }^{32}$ R. Raj, S. C. Maroo, and E. N. Wang, Nano Lett. 13, 1509-1515 (2013).

${ }^{33}$ M. Y. Zhao, X. P. Yang, and X. N. Yang, Acta Phys. Chim. Sin. 31, 1489-1498 (2015).
${ }^{34}$ J. Marti, J. Sala, and E. Guardia, J. Mol. Liq. 153, 72-78 (2010).

${ }^{35}$ J. Marti, G. Nagy, M. C. Gordillo, and E. Guardia, J. Chem. Phys. 110, 23987-23994 (2006).

${ }^{36}$ G. Cicero, J. C. Grossman, E. Schwegler, F. Gygi, and G. Galli, J. Am. Chem. Soc. 130, 1871-1878 (2008).

${ }^{37}$ C. Sendner, D. Horinek, L. Bocquet, and R. R. Netz, Langmuir 25, 10768-10781 (2009).

${ }^{38}$ M. C. Gordillo and J. Marti, Chem. Phys. Lett. 329, 341-345 (2000).

${ }^{39}$ W. H. Noon, K. D. Ausman, R. E. Smalley, and J. Ma, Chem. Phys. Lett. 355, 445-448 (2002).

${ }^{40}$ K. Koga, G. T. Gao, H. Tanaka, and X. C. Zeng, Nature 412, 802-805 (2001).

${ }^{41}$ G. Hummer, J. C. Rasaiah, and J. P. Noworyta, Nature 414, 188-190 (2001).

${ }^{42}$ M. K. Rana and A. Chandra, J. Chem. Phys. 138, 204702 (2013).

${ }^{43}$ J. A. Thomas, A. J. H. McGaughey, and O. Kuter-Arnebeck, Int. J. Therm. Sci. 49, 281-289 (2010)

${ }^{44}$ W. L. Jorgensen, J. Chandrasekhar, J. D. Madura, R. W. Impey, and M. L. Klein, J. Chem. Phys. 79, 926-935 (1983).

${ }^{45}$ T. S. Mahadevan and S. H. Garofalini, J. Phys. Chem. B 111, 8919-8927 (2007).

${ }^{46}$ H. J. C. Berendsen, J. R. Grigera, and T. P. Straatsma, J. Phys. Chem. 91, 6269-6271 (1987).

${ }^{47}$ P. Mark and L. Nilsson, J. Phys. Chem. A 105, 9954-9960 (2001).

${ }^{48}$ M. C. Gordillo and J. Marti, J. Chem. Phys. 117, 3425-3430 (2002).

${ }^{49}$ T. Werder, J. H. Walther, R. L. Jaffe, T. Halicioglu, and P. Koumoutsakos, J. Phys. Chem. B 107, 1345-1352 (2003)

${ }^{50}$ J. Marti and M. C. Gordillo, J. Chem. Phys. 119, 12540-12546 (2003).

${ }^{51}$ M. C. Gordillo, G. Nagy, and J. Marti, J. Chem. Phys. 123, 054707 (2005).

${ }^{52}$ N. Marković, P. U. Andersson, M. B. Någård, and J. B. Pettersson, Chem. Phys. 247, 413-430 (1999).

${ }^{53}$ T. Werder, J. H. Walther, R. L. Jaffe, T. Halicioglu, F. Noca, and P. Koumoutsakos, Nano Lett. 1, 697-702 (2001).

${ }^{54}$ G. Scocchi, D. Sergi, C. D'Angelo, and A. Ortona, Phys. Rev. E 84, 061602 (2011).

${ }^{55}$ Y. B. Wu and N. R. Aluru, J. Phys. Chem. B 117, 8802-8813 (2013).

${ }^{56}$ M. J. Bojan and W. A. Steele, Langmuir 3, 1123-1127 (1987).

${ }^{57}$ F. M. Fowkes and W. D. Harkins, J. Am. Chem. Soc. 62, 3377-3386 (1940).

${ }^{58}$ G. R. Jenness, O. Karalti, and K. D. Jordan, Phys. Chem. Chem. Phys. 12, 6375-6381 (2010).

${ }^{59}$ A. Alexiadis and S. Kassinos, Chem. Rev. 108, 5014-5034 (2008).

${ }^{60}$ J. S. Kim et al., Sci. Rep. 3, 2309 (2013).

${ }^{61}$ N. Choudhury and B. M. Pettitt, J. Phys. Chem. B 109, 6422-6429 (2005).

${ }^{62}$ R. J. Mashl, S. Joseph, N. R. Aluru, and E. Jakobsson, Nano Lett. 3, 589-592 (2003).

${ }^{63}$ Y. Liu, Q. Wang, T. Wu, and L. Zhang, J. Chem. Phys. 123, 234701 (2005).

${ }^{64}$ I. Hanasaki and A. Nakatani, J. Chem. Phys. 124, 174714 (2006).

${ }^{65}$ S. L. Mayo, B. D. Olafson, and W. A. Goddard, J. Phys. Chem. 94, 8897-8909 (1990).

${ }^{66}$ J. A. Elliott, S. Hanna, A. M. S. Elliott, and G. E. Cooley, Phys. Chem. Chem. Phys. 1, 4855-4863 (1999).

${ }^{67}$ S. T. Cui and S. J. Paddison, J. Phys. Chem. C 119, 12848-12855 (2015).

${ }^{68}$ J.-P. Ryckaert, G. Ciccotti, and H. J. Berendsen, J. Comput. Phys. 23, 327-341 (1977)

${ }^{69}$ S. Plimpton, J. Comput. Phys. 117, 1-19 (1995).

${ }^{70}$ C. J. Fennell and J. D. Gezelter, J. Chem. Phys. 124, 234104 (2006).

${ }^{71}$ G. S. Hwang, M. Kaviany, J. T. Gostick, B. Kientiz, A. Z. Weber, and M. H. Kim, Polymer 52, 2584-2593 (2011).

${ }^{72}$ R. Rowley and M. Painter, Int. J. Thermophys. 18, 1109-1121 (1997).

${ }^{73}$ D. J. Evans and B. L. Holian, J. Chem. Phys. 83, 4069-4074 (1985).

${ }^{74}$ M. Zokaie and M. Foroutan, RSC Adv. 5, 39330-39341 (2015).

${ }^{75}$ X. Xia, L. Perera, U. Essmann, and M. L. Berkowitz, Surf. Sci. 335, 401-415 (1995).

${ }^{76}$ N. Choudhury and B. M. Pettitt, J. Am. Chem. Soc. 127, 3556-3567 (2005). 